\title{
EFEITOS DO CPPU E DO ÁCIDO GIBERÉLICO NAS CARACTERÍSTICAS DOS CACHOS DA UVA DE MESA 'CENTENNIAL SEEDLESS'
}

\author{
ERASMO JOSÉ PAIOLI PIRES \\ RENATO VASCONCELOS BOTELHO ${ }^{2}$ \\ MAURILO MONTEIRO TERRA ${ }^{3}$
}

\begin{abstract}
RESUMO - Foram conduzidos dois experimentos em vinhedo comercial, na região de Jundiaí-SP, com o objetivo de se estudar os efeitos do CPPU e do $\mathrm{AG}_{3}$ nas características dos cachos e dos bagos de uvas 'Centennial Seedless'. No primeiro experimento, utilizou-se de CPPU a $0 ; 5,0 ; 7,5 ; 10,0 ; 12,5 ; 15,0$ e $17,5 \mathrm{mg} . \mathrm{L}^{-1}$. No segundo ensaio, empregou-se $\mathrm{AG}_{3}$ nas doses de $0,5,10$, $15,20,25$ e $30 \mathrm{mg} \cdot \mathrm{L}^{-1}$. Tanto o CPPU como o $\mathrm{AG}_{3}$ aumentaram a massa e largura dos cachos; a massa, comprimento e largura dos bagos, e o diâmetro dos pedice-
\end{abstract}

los. O aumento do comprimento dos cachos só foi significativo pela aplicação de $\mathrm{AG}_{3}$. Ambos os reguladores de crescimento reduziram o teor de sólidos solúveis totais do mosto. Aplicações de CPPU e $\mathrm{AG}_{3}$ a $5 \mathrm{mg} . \mathrm{L}^{-1}$ proporcionaram aumento da massa dos bagos em 59,0 e $78,7 \%$, respectivamente, e formação de cachos soltos, com pedicelos mais flexíveis. Doses superiores levaram à formação de cachos muito grandes, excessivamente compactos, com pedicelos rijos e muito engrossados e, portanto, de valor comercial reduzido.

TERMOS PARA INDEXAÇÃO: Videira, Vitis vinifera L., regulador de crescimento, citocinina, giberelina.

\section{EFFECTS OF CPPU AND GIBBERELLIC ACID ON THE CLUSTERS CHARACTERISTICS OF 'CENTENNIAL SEEDLESS' TABLE GRAPE}

\begin{abstract}
Two trials were conducted in a commercial vineyard, in the region of Jundiaí-SP, with the objective of studying the effects of CPPU and $\mathrm{GA}_{3}$ on the clusters and berries characteristics of 'Centennial Seedless' table grape. In the first experiment, it was used CPPU at $0 ; 5.0 ; 7.5 ; 10.0 ; 12.5$; 15.0 and $17.5 \mathrm{mg} . \mathrm{L}^{-1}$. In a second trial, $\mathrm{GA}_{3}$ was used at the doses of: $0,5,10,15,20,25$ e $30 \mathrm{mg}^{-L^{-1}}$. Both, CPPU and $\mathrm{GA}_{3}$ increased the clusters weight and width; the berries weight, length and width; and the pedicel
\end{abstract}

diameter. The increase in cluster length was significant only for $\mathrm{GA}_{3}$ applications. Both growth regulators decreased the total soluble solids content. Applications of CPPU and $\mathrm{GA}_{3}$ at $5 \mathrm{mg} . \mathrm{L}^{-1}$, provided increases in berries weight in 59.0 and $78.7 \%$, respectively, and formation of untied clusters, with flexible pedicels. Higher doses led to formation of clusters too much large, excessively compact, with pedicels very thick, and, therefore, with reduced commercial value.

INDEX TERMS: Vine, Vitis vinifera L., growth regulator, cytokinin, gibberellin.

\section{INTRODUÇÃO}

As giberelinas são os reguladores de crescimento mais amplamente utilizados em nível comercial na Viticultura, visando principalmente ao aumento do tamanho e da fixação dos bagos, à descompactação dos cachos e à eliminação de sementes. Segundo Pires et al. (1986), as respostas às aplicações de ácido giberélico variam em função da cultivar e das condições de cultivo.
Para a cultivar sem sementes Sultanina, El Hodairi et al. (1995) constataram aumento da massa dos cachos e comprimento dos bagos, pela aplicação de $\mathrm{AG}_{3}$ a $50 \mathrm{mg} . \mathrm{L}^{-1}$, após a frutificação. Resultados semelhantes foram obtidos por Retamales et al. (1997) em uvas 'Sultanina', mediante duas aplicações, em pós-florescimento, de $\mathrm{AG}_{3}$ ou mistura de giberelinas $\left(\mathrm{AG}_{3}, \mathrm{AG}_{1}, \mathrm{AG}_{4}\right.$ e $\left.\mathrm{AG}_{7}\right)$ a $40 \mathrm{mg} . \mathrm{L}^{-1}$.

1. Engenheiro Agrônomo, Dr. Instituto Agronômico de Campinas (IAC), Caixa Postal 28, 13001-970 -Campinas, SP, bolsista CNPq.

2. Engenheiro Agrônomo, Dr. IAC, Bolsista FAPESP.

3. Engenheiro Agrônomo, Dr. IAC, Bolsista CNPq. 
Entretanto, para Retamales et al. (1995) a busca de um substituto para o ácido giberélico é importante tendo em vista que este apresenta alguns efeitos indesejáveis como: redução da fertilidade de gemas, aumento do vigor das plantas, degrana dos cachos pós-colheita e maior suscetibilidade dos frutos às podridões.

O CPPU (N-(2-cloro-piridil)-N-feniluréia), também denominado forchlorofenuron, é reconhecido como uma citoninina do grupo da feniluréias, muito mais potente que outras citocininas derivadas da adenina, apresentando baixíssima toxicidade tanto para plantas como para animais, e para a toxicidade dermal aguda, é necessário dosagem superior a 2000 mg. $\mathrm{kg}^{-1}$ (Nickel, 1986).

Em fruticultura, o CPPU promove o crescimento dos frutos de kiwis, pêras, uvas e maçãs (Arima et al., 1995), e sua ação está relacionada ao aumento da divisão celular (Ben Arie et al., 1997). Também promove o pegamento dos frutos de melões (Arima et al., 1995) e o raleamento de frutos de maçã (Greene, 1989).

Em trabalho realizado com as cultivares de videiras apirenas Sovereign Coronation e Summerland Selection 495, Reynolds et al. (1992) verificaram que aplicações de CPPU nas doses de 0,1 e $10 \mathrm{mg} . \mathrm{L}^{-1}$, quando os bagos atingiram $5 \mathrm{~mm}$ de diâmetro, aumentaram linearmente a massa dos cachos e bagos, o número de bagos, o comprimento dos engaços e a acidez titulável total do mosto. Constatou-se também redução linear para o teor de sólidos solúveis totais, $\mathrm{pH}$ e antocianinas totais. Outros autores também relataram efeitos do CPPU no aumento do tamanho dos bagos em diferentes cultivares de videira (Ben Arie et al., 1997; Leão et al., 1999; Miele et al., 2000).

Realizou-se este trabalho com o objetivo de auferir os efeitos de CPPU e $\mathrm{AG}_{3}$ nas características dos cachos da uva de mesa 'Centennial Seedless', tendo em vista as poucas informações sobre os efeitos de reguladores de crescimento nessa cultivar.

\section{MATERIAL E MÉTODOS}

Os experimentos foram instalados em vinhedo comercial da cultivar Centennial Seedless, localizado no município de Jundiaí-SP, com coordenadas geográficas de $23^{\circ} 06^{\prime} \mathrm{S}$ e $46^{\circ} 55^{\prime} \mathrm{O}$, e altitude de $715 \mathrm{~m}$. As videiras, enxertadas sobre porta-enxerto 420-A, em primeiro ano de produção, foram conduzidas no sistema de pérgula, no espaçamento de $5 \times 3 \mathrm{~m}$. Todas as práticas culturais, exceto a utilização de ácido giberélico, foram idênticas ao sistema convencional da propriedade, para toda a área experimental.

No primeiro experimento, utilizou-se do CPPU nas doses de $0 ; 5,0 ; 7,5 ; 10,0 ; 12,5 ; 15,0$ e 17,5 mg.L ${ }^{-1}$.
No segundo ensaio, empregou-se $\mathrm{AG}_{3}$ nas doses de 0,5 , $10,15,20,25$ e $30 \mathrm{mg} . \mathrm{L}^{-1}$.

Os reguladores de crescimento foram aplicados mediante única aplicação por imersão dos cachos em solução aquosa contida em recipiente plástico, 14 dias após o pleno florescimento. Para os tratamentos com $\mathrm{AG}_{3}$, foi adicionado à solução espalhante adesivo, marca comercial Hiraragen- $S^{\circledR}$, a $0,1 \%$.

$\mathrm{O}$ delineamento experimental foi em blocos casualizados com 7 tratamentos, 6 repetições e parcelas constituídas por três cachos. Videiras semelhantes em vigor foram selecionadas para todos os tratamentos em cada bloco.

A coleta dos cachos foi feita no ponto de colheita comercial, isto é, quando a testemunha atingiu o teor de sólidos solúveis totais de $14,0^{\circ}$ Brix, o que ocorreu em 4 de janeiro de 2001. Posteriormente, os cachos foram acondicionados em sacos de polietileno devidamente identificados e levados para laboratório para a realização das avaliações.

Para cada cacho das parcelas, foram avaliadas as seguintes variáveis: massa dos cachos e bagos (subamostra de 20 bagos), em balança de precisão; comprimento e largura de cachos e bagos (subamostra de 20 bagos) e diâmetro dos pedicelos, com paquímetro digital, e teor de sólidos solúveis totais do mosto, utilizando-se de refratômetro de mesa ATAGO-3T, com autocompensação de temperatura (Lutz, 1985).

Os resultados obtidos foram submetidos à análise de variância e estudo de regressão polinomial.

\section{RESULTADOS E DISCUSSÃO}

Para as variáveis massa e largura dos cachos (Figuras 1 e 3); massa, largura, comprimento dos bagos (Figuras 4, 5, 6), e diâmetro dos pedicelos (Figura 7), verificou-se significância para regressão quadrática tanto para doses de CPPU como de $\mathrm{AG}_{3}$. Em relação ao comprimento dos cachos, somente houve significância para regressão quadrática para doses de $\mathrm{AG}_{3}$ (Figura 2).

As doses estimadas para a maior massa dos cachos foram de 12,2 e 19,3 mg. $\mathrm{L}^{-1}$, para o CPPU e o $\mathrm{AG}_{3}$, respectivamente (Figura 1). Efeitos do CPPU no aumento da massa dos cachos também foram verificados por Reynolds et al. (1992), em uvas sem sementes 'Sovereign Coronation' e 'Summerland Selection 495'. Resultados semelhantes foram verificados por El Hodairi et al. (1995) para a cultivar apireno Sultanina, mediante aplicação de $\mathrm{AG}_{3}$ a 50mg.L $\mathrm{L}^{-1}$, após a frutificação.

Para a máxima massa dos bagos, as doses estimadas foram de 12,6 e 20,7 mg. $\mathrm{L}^{-1}$, para CPPU e

Ciênc. agrotec., Lavras. V.27, n.2, p.305-311, mar./abr., 2003 
$\mathrm{AG}_{3}$, respectivamente (Figura 4). Efeitos do CPPU no aumento da massa, comprimento e largura dos bagos foram relatados por vários autores em diferentes cultivares de uva sem sementes (Nickel, 1986; Rey- nolds et al., 1992; Retamales et al., 1995; Ben Arie et al., 1997), assim como de aplicações de $\mathrm{AG}_{3}$ (El Hodairi et al., 1995; Retamales et al., 1997).

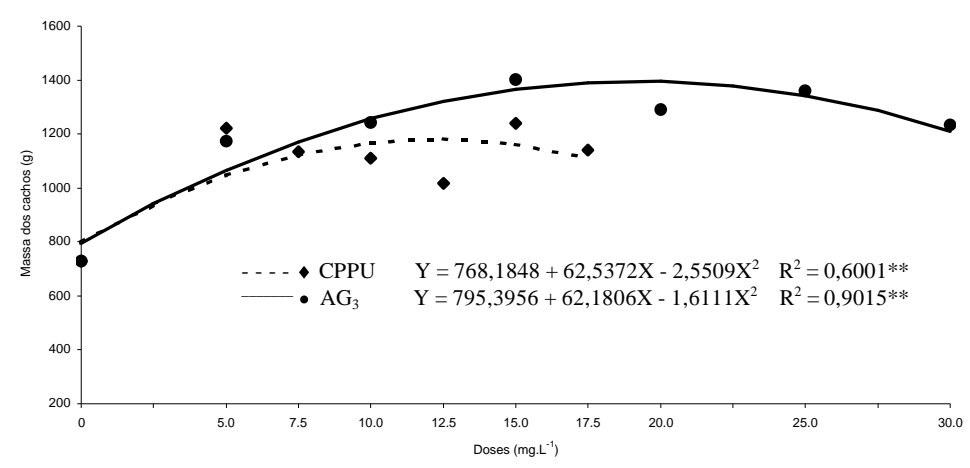

FIGURA 1 - Massa dos cachos (g) de uvas da cultivar Centennial Seedless tratados com $\mathrm{AG}_{3}$ e CPPU - Jundiaí-SP, 2001.

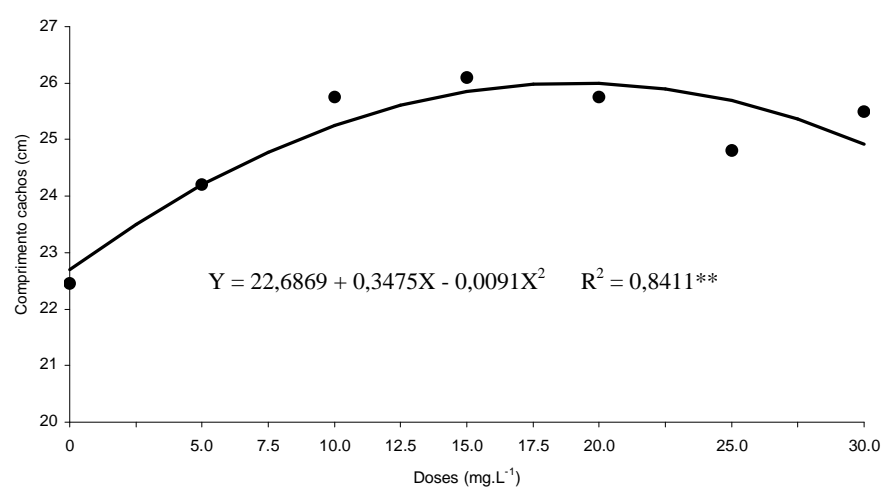

FIGURA 2 - Comprimento dos cachos $(\mathrm{cm})$ de uvas da cultivar Centennial Seedless tratados com $\mathrm{AG}_{3}$ - JundiaíSP, 2001. 


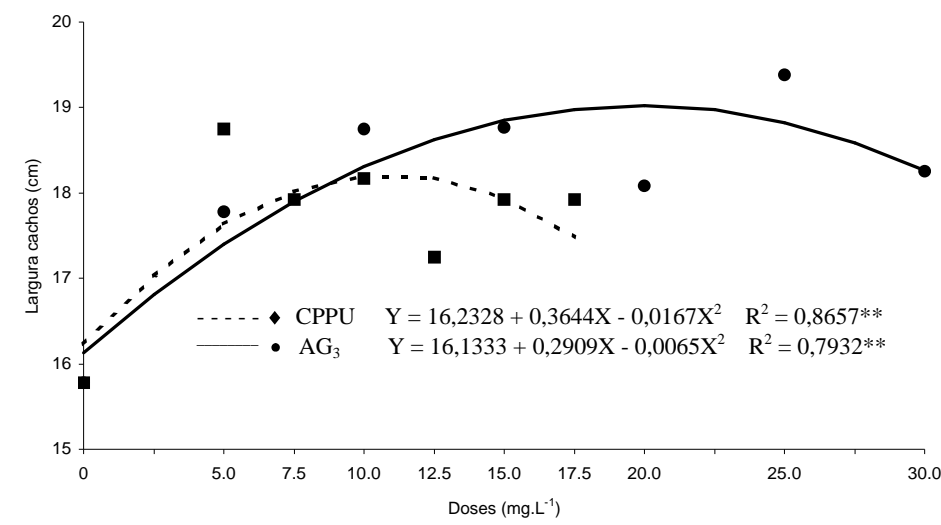

FIGURA 3 - Largura dos cachos $(\mathrm{cm})$ de uvas da cultivar Centennial Seedless tratados com $\mathrm{AG}_{3}$ e CPPU - JundiaíSP, 2001.

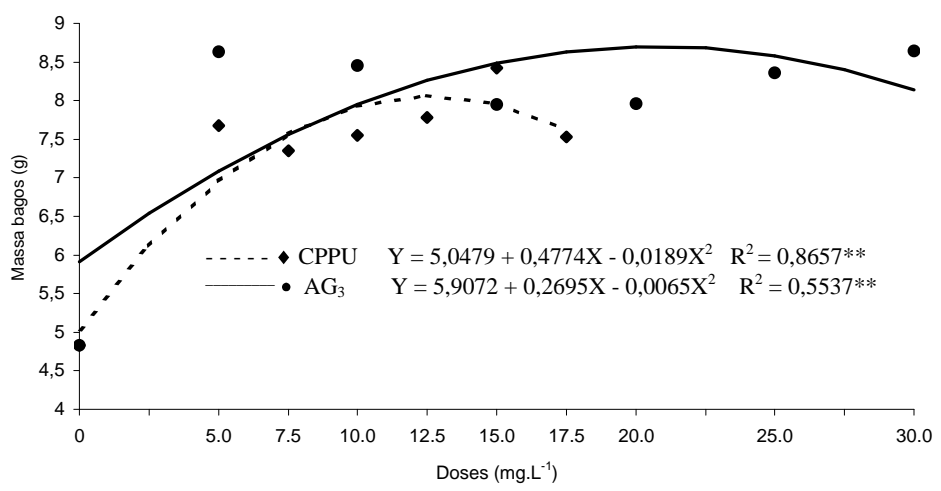

FIGURA 4 - Massa dos bagos (g) de uvas da cultivar Centennial Seedless tratadas com $\mathrm{AG}_{3}$ e CPPU - Jundiaí-SP, 2001.

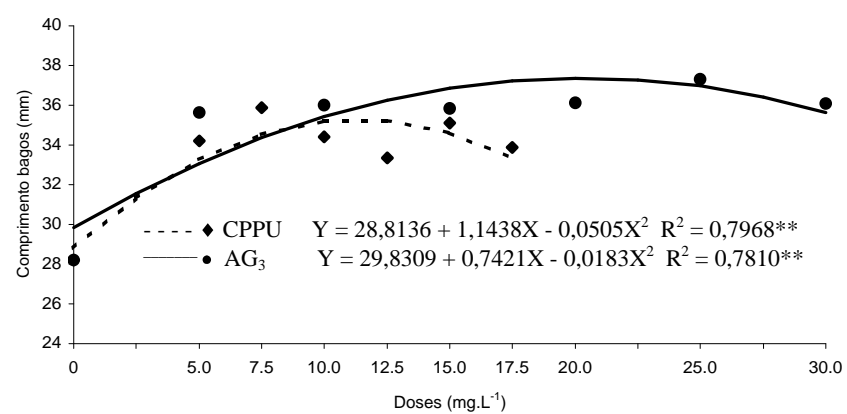

FIGURA 5 - Comprimento dos bagos $(\mathrm{mm})$ de uvas da cultivar Centennial Seedless tratadas com $\mathrm{AG}_{3}$ e CPPU Jundiaí-SP, 2001.

Ciênc. agrotec., Lavras. V.27, n.2, p.305-311, mar./abr., 2003 




FIGURA 6 - Largura dos bagos (mm) de uvas da cultivar Centennial Seedless tratadas com $\mathrm{AG}_{3}$ e CPPU - JundiaíSP, 2001.

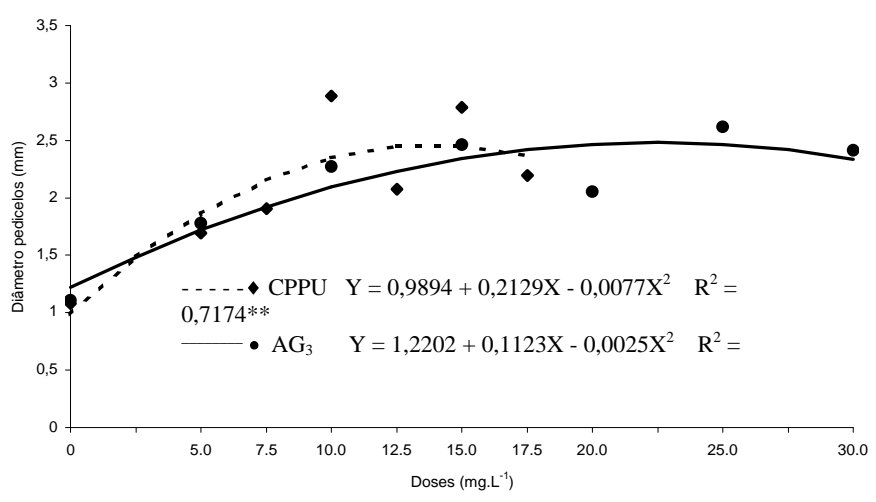

FIGURA 7 - Diâmetro dos pedicelos $(\mathrm{mm})$ de uvas da cultivar Centennial Seedless tratadas com $\mathrm{AG}_{3}$ e CPPU Jundiaí-SP, 2001.

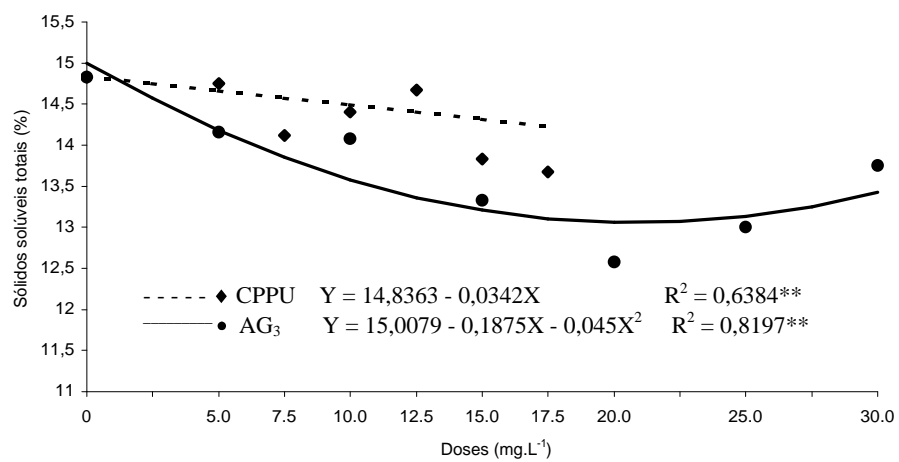

FIGURA 8 - Teor de sólidos solúveis totais (\%) do mosto de uvas da cultivar Centennial Seedless tratadas com $\mathrm{AG}_{3}$ e CPPU (Jundiaí-SP, 2001). 
Segundo Métraux (1988), o crescimento de órgãos vegetais promovidos por giberelinas deve-se principalmente a um aumento do tamanho de células já existentes ou recentemente divididas. Porém, esse crescimento das células pode ser acompanhado por um incremento do número de células. Citocininas também podem promover o desenvolvimento de órgãos vegetais mediante estímulo da divisão celular (McGraw, 1988).

$\mathrm{O}$ aumento do diâmetro dos pedicelos (Figura 7) promovido pelas aplicações dos reguladores de crescimento está de acordo com os relatos de outros autores. Retamales et al. (1995) observaram que os cachos de uvas 'Thompson Seedless' tratadas com CPPU apresentavam os pedicelos e ráquis lignificados e engrossados. Cooper et al. (1993) também apresentaram relatos semelhantes para uvas tratadas com $\mathrm{AG}_{3}$. Segundo esses autores, esse efeito leva a uma redução da flexibilidade dos pedicelos, provocando o aumento da degrana póscolheita dos cachos.

Para a variável teor de sólidos solúveis totais, verificou-se significância para regressão linear para doses de CPPU e regressão quadrática para doses de $\mathrm{AG}_{3}(\mathrm{Fi}-$ gura 8). Esses resultados demonstraram que esses reguladores de crescimento apresentam efeito no atraso da maturação dos cachos. Leão et al. (1999) verificaram atraso de 8 dias na maturação dos cachos de uvas da cultivar Perlette tratadas com CPPU, ao passo que Retamales et al. (1995) observaram que houve atraso de até 21 dias em uvas da cultivar Thompson Seedless. Redução do teor de sólidos solúveis totais de cachos tratados com $\mathrm{AG}_{3}$ também foi constatada por Maraschin et al. (1986) em uvas 'Niagara Branca'.

$\mathrm{O}$ fato de que citocininas e giberelinas retardam a maturação de frutos já é bastante conhecido, porém seus mecanismos de ação ainda não foram totalmente esclarecidos (Ludford, 1988). Segundo Chitarra \& Chitarra (1990), o retardo da senescência tem sido associado à redução da taxa de perda de proteínas e RNA. As citocininas parecem atuar nesse processo, suprimindo a síntese de proteases e prevenindo qualquer aumento na atividade da RNAase.

Embora tenha se constatado, pela análise de regressão, que as doses para as máximas massas dos cachos e de bagos situaram-se próximo a $12,5 \mathrm{mg} . \mathrm{L}^{-1}$ e 20 mg. $\mathrm{L}^{-1}$, para CPPU e $\mathrm{AG}_{3}$, respectivamente, do ponto de vista agronômico e de comercialização, nessas doses, os cachos ficaram muito grandes, demasiadamente compactos, com os pedicelos e engaços engrossados e muito rijos. Por outro lado, a dose de $5 \mathrm{mg} . \mathrm{L}^{-1}$, tanto para CPPU como para $\mathrm{AG}_{3}$, possibilitou a formação de cachos mais soltos, com pedicelos mais flexíveis e bagos de bom tamanho. Nessa dose, os aumentos na massa dos bagos foram de 59,0 e 78,7\% em relação à testemunha, com média de massa dos cachos de 1220 e $1174 \mathrm{~g}$, para CPPU e $\mathrm{AG}_{3}$, respectivamente.

$\mathrm{O}$ atraso na maturação dos cachos promovido pela aplicação dos reguladores de crescimento pode ser um efeito interessante para o viticultor, visando principalmente ao escalonamento da colheita e à comercialização dos frutos em períodos de menor oferta, quando os preços são superiores.

\section{CONCLUSÕES}

a) Aplicações de CPPU e $\mathrm{AG}_{3}$ aumentaram a massa e as dimensões dos cachos e bagos; o diâmetro dos pedicelos reduziu o teor de sólidos solúveis totais do mosto de uvas 'Centennial Seedless'.

b) Agronomicamente, CPPU ou $\mathrm{AG}_{3}$ a $5 \mathrm{mg} . \mathrm{L}^{-1}$ foram os tratamentos que apresentaram os melhores resultados, conferindo aumento da massa dos bagos entre 59,0 e $78,7 \%$, e formação de cachos soltos com pedicelos flexíveis.

\section{AGRADECIMENTOS}

Ao Sr. Valdeir Biudes Hermoso, pelo apoio e colaboração na condução deste experimento.

\section{REFERÊNCIAS BIBLIOGRÁFICAS}

ARIMA, Y.; OSHIMA, K.; SHUDO, K. Evolution of a novel urea-type cytokinin: Horticultural uses of forchlorofenuron. Acta Horticulturae, Leiden, n. 394, p. $75-83,1995$.

BEN ARIE, R.; SARIG, P.; COHEN AHDUT, Y.; SONEGO, L.; KAPULONOV, T.; LISKER, N. CPPU and $\mathrm{GA}_{3}$ effects on pre- and post-harvest quality of seedless and seeded grapes. Acta Horticulturae, Leiden, n. 463, p. 349-357, 1997.

CHITARRA, M. I. F.; CHITARRA, A. B. Aspectos fisiológicos do desenvolvimento dos frutos. In:

Pós-colheita de frutos e hortaliças: fisiologia e manuseio. Lavras: ESAL-FAEPE, 1990. p. 23-64.

COOPER, T.; RETAMALES, J.; BANGERTH, F. Berry drop occurence as affected by $\mathrm{GA}_{3}$ and promalin applications in Thompson Seedless grapes. Acta Horticulturae, Leiden, n. 329, p. 134-136, 1993. 
EL HODAIRI, M. H.; IBRAHIM, S. B.; AL BASHIR, A. H.; AL BARKOULI, A. A.; HUSSEIN, A. R. Effect of gibberellic acid on Sultanine Seedless grape variety grown in the Libyan Sahara. Acta Horticulturae, Leiden, n. 409, p. 93-97, 1995.

GREENE, D. W. Regulation of fruit set in tree fruits with plant growth regulators. Acta Horticulturae, Leiden, n. 239, p. 323-333, 1989.

LEÃO, P. C. S.; LINO JÚNIOR, E. C.; SANTOS, E. S. Efeitos do CPPU e ácido giberélico sobre o tamanho de bagas de uvas Perlette cultivada no Vale do São Francisco. Revista Brasileira de Fruticultura, Jaboticabal, v. 21, n. 1, p. 74-78, 1999.

LUDFORD, P. M. Postharvest hormones changes in vegetables and fruits. In: DAVIES, P. J. Plant hormones and their role in plant growth and development. 2. ed. Dordrecht: Kluwer Academic, 1988.

LUTZ, A. Normas analíticas do Instituto Adolfo Lutz: métodos químicos para análise de alimentos. 3. ed. São Paulo: Coordenadoria dos Serviços Técnicos Especializados-SES, 1985. 533 p.

MACGRAW, B. A. Cytokinin biosynthesis and metabolism. In: DAVIES, P. J. Plant hormones and their role in plant growth and development. 2. ed. Dordrecht: Kluwer Academic, 1988. p. 76-93.

MARASCHIN, M.; GUERRA, M. P.; SILVA, A. L. Efeitos do ácido giberélico e ethephon sobre as características dos cachos e frutos da cv. Niagara Branca (Vitis labrusca L.). Revista Brasileira de Fruticultura, Cruz das Almas, v. 8, n. 2, p. 51-57, 1986.

MÉTRAUX, J. P. Gibberellins and plant cell elongation. In: DAVIES, P. J. Plant hormones and their role in plant growth and development. 2. ed. Dordrecht: Kluwer Academic, 1988. p. 296-317.

MIELE, A.; RIZZON, L. A.; DALL'AGNOL, I. Efeito de reguladores de crescimento no tamanho da baga e na composição do mosto da uva 'Itália'. Revista Brasileira de Fruticultura, Jaboticabal, v. 22, n. 2, p. 272-276, 2000.

NICKEL, L. G. The effects of N-(2-chloro-4-pyridil)$\mathrm{N}$ '-phenylurea and the 2-chloro-benzyl ester of dicamba on the growth and sugar contents of grapes. Acta Horticulturae, Leiden, n. 179, p. 805-806, 1986

PIRES, E. J. P.; FAHL, J. I.; CARELLI, M. L. C.; TERRA, M. M.; PASSOS, I. R. S.; CRUZ, L. S. P.; MARTINS, F. P. Respostas a aplicação de ácido giberélico (GA) em panículas de videira do cultivar IAC 871-13 A Dona. In: CONGRESSO BRASILEIRO DE FRUTICULTURA, 8., 1986, Brasília. Anais... Brasília: Embrapa DDT/CNPq, 1986. v. 2, p. 473-477.

REYNOLDS, A. G.; WARDLE, D. A.; ZUROWSKI, C.; LOONEY, N. E. Phenylureas CPPU and thidiazuron affect yield components, fruit composition, and storage potential of four seedless grape selections. Journal of the American Society for Horticultural Science, Alexandria, v. 117, p. 85-89, 1992.

RETAMALES, J.; BANGERTH，F.; COOPER，T. CALLEJAS, R. Effects of CPPU and $\mathrm{GA}_{3}$ on fruit quality of Sultanina table grape. Acta Horticulturae, Leiden, n. 394, p. 149-157, 1995.

RETAMALES, J.; RIVAS, A.; PINTO, M. A novel mixture of gibberelins can replace both $\mathrm{GA}_{3}$ and CPPU on Thompson Seedless grapes. Acta Horticulturae, Leiden, n. 463, p. 219-224, 1997. 